\title{
Distinct Somatic Alteration Features Identified by Gene Panel Sequencing in Korean Triple-Negative Breast Cancer with High Ki67 Expression
}

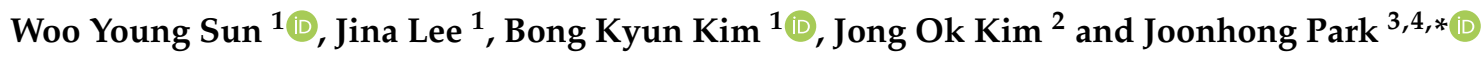 \\ 1 Department of Surgery, College of Medicine, The Catholic University of Korea, Seoul 06591, Korea; \\ sun2729@catholic.ac.kr (W.Y.S.); ljatempe@naver.com (J.L.); bios777@naver.com (B.K.K.) \\ 2 Department of Pathology, College of Medicine, The Catholic University of Korea, Seoul 06591, Korea; \\ jkim@catholic.ac.kr \\ 3 Department of Laboratory Medicine, Jeonbuk National University Medical School and Hospital, \\ Jeonju 54907, Korea \\ 4 Clinical Medicine-Biomedical Research Institute, Jeonbuk National University Hospital, Jeonju 54907, Korea \\ * Correspondence: miziro@jbnu.ac.kr; Tel.: +82-63-250-1218
}

Citation: Sun, W.Y.; Lee, J.; Kim, B.K.; Kim, J.O.; Park, J. Distinct Somatic Alteration Features Identified by Gene Panel Sequencing in Korean Triple-Negative Breast Cancer with High Ki67 Expression. Diagnostics 2021, 11, 416. https://doi.org/ 10.3390/diagnostics11030416

Academic Editors: Moira Ragazzi and Dario de Biase

Received: 6 January 2021

Accepted: 28 January 2021

Published: 1 March 2021

Publisher's Note: MDPI stays neutral with regard to jurisdictional claims in published maps and institutional affiliations.

Copyright: (c) 2021 by the authors. Licensee MDPI, Basel, Switzerland. This article is an open access article distributed under the terms and conditions of the Creative Commons Attribution (CC BY) license (https:// creativecommons.org/licenses/by/ $4.0 /)$.

\begin{abstract}
This study aimed to clarify the genetic difference between Korean triple-negative breast cancer (TNBC) and other breast cancer (BC) subtypes. TNBC was defined as the absence of hormonal receptors and human epidermal growth factor receptor 2 (HER2) amplification. DNA panel of the Ion Torrent Oncomine Comprehensive Assay (OCA) v3 was performed to identify somatic alteration in 48 specimens. In a total of 102 alterations (37 nonsense, 35 missense, 8 frameshift and 22 amplifications), 30 nucleotide alterations ( 24 nonsense, 1 missense, and 5 frameshift) were newly identified. The eight most commonly altered genes were PIK3CA, TP53, ERBB2, BRCA2, FANCD2, $A K T 1, B R C A 1$, and FANCA. TNBC had significantly lower mutation frequency in PIK3CA (TNBC vs. hormone receptor-positive and HER2-negative $\mathrm{BC}$ [HRPBC], $p=0.009$ ), but higher mutation frequency in TP53 (TNBC vs. HRPBC, $p=0.036$; TNBC vs. hormone receptor-positive and HER2positive $\mathrm{BC}[\mathrm{HHPBC}], p=0.004)$. TNBC showed frequently higher Ki-67 expression than any positive BC $(p=0.004)$ due to HRPBC $(p<0.001)$. TNBC with high Ki-67/unmutated PIK3CA/mutated TP53 appears at a younger age (52.2 \pm 7.6 years), compared to other subtypes ( $63.7 \pm 11.0$ years). TNBC with high $\mathrm{Ki}-67 /$ unmutated $P I K 3 C A$ /mutated TP53 may be related to relatively early onset BC. These findings demonstrate the genomic heterogeneity between TNBC and other BC subtypes and could present a new approach for molecular targeted therapy in TNBC patients.
\end{abstract}

Keywords: triple-negative breast cancer; early detection; Ki-67; PIK3CA; TP53; gene panel sequencing

\section{Introduction}

Breast cancer (BC) is the most frequently diagnosed cancer $(11.6 \%)$ and the leading cause of cancer death (6.6\%) among women worldwide [1]. In Korea, 22,395 invasive BC cases were recorded in 2017, making it the fifth most frequent cancer type (9.6\%) [2]. Hormone receptor (HR)-positive and human epidermal growth factor receptor 2 (HER2)negative BC (HRPBC) was the most common (65.9\%), followed by HR-positive and HER2positive BC (HHPBC) (11.7\%), HR-negative and HER2-positive BC (HER2PBC, 10.2\%), and HR-negative and HER2-negative BC (TNBC, 12.2\%) [2]. BC is recognized as a complex and diverse group of neoplastic diseases of the breast with distinct molecular characteristics and clinical outcomes [3]. With the elucidation of the intrinsic subtypes of BC, targeted therapies including endocrine therapy or anti-HER2 therapy have been tailored to the specific pathophysiology of HRPBC or HER2PBC [4]. However, patients with TNBC are not eligible for effective selective hormonal modulators or anti-HER2 therapy. The lack of expression of HR and HER2 amplification in TNBC makes it an orphan disease when considering standard therapeutic regimens for BC. An alternative classification divides TNBC into 
basal-like 1 (BL1), BL2, mesenchymal, and luminal androgen receptor [5]. Even though these subgroups can be further stratified through multi-omics approaches [6], they are largely unknown. Inherited mutations in the $B R C A 1$ and $B R C A 2$ genes as tumor suppressor lead to basal-like BC [7], and many TNBC with intact $B R C A 1 / 2$ are classified as BRCAness lesions [8]. Combined loss of the TP53 was identified in 30 to $40 \%$ of sporadic TNBC [9]. Additional alterations include PIK3CA mutation or PTEN loss and/or enhanced EGFR, WNT and MYC signaling. TP53, PTEN, and RB1 are also the most frequent drivers of metastasis in diverse types of human solid cancers including breast cancer [10]. TNBC has been known to be related with a worse prognosis and lower survival rate than other BC subtypes [11]. Even though PARP inhibitors have been approved for chemotherapy of BRCA1/2 mutant/BRCAness TNBC, emergence of clones that resist PARP inhibition through multiple mechanisms is a crucial clinical problem [12]. Considerable racial variations in clinical manifestation and incidence of TNBC have been reported, likely due to the diverse nature of the disease [5,13]. Moreover, the histologic differences of TNBC as well as the heterogeneity in the clinical manifestation may be related to variations in molecular background [14].

The present study aimed to compare the mutation characteristics of $\mathrm{BC}$ among its subtypes to clarify the genetic difference between TNBC and other BC subtypes in the Korean population, using comprehensive cancer panel sequencing.

\section{Materials and Methods}

\subsection{Patients and Specimens}

We evaluated 48 patients diagnosed with primary BC and treated at the Department of Surgery, Daejeon St. Mary's Hospital (Daejeon, Korea) between January 2015 and December 2019. Primary tumor tissues and lymph nodes were surgically resected as standard institutional treatment, and specimens were examined by a board-certified pathologist. The tissues were dissected for histological diagnosis and immunohistochemistry (IHC) staining and then archived as formalin-fixed paraffin-embedded (FFPE) specimens. IHC staining was performed to determine estrogen receptor (ER), progesterone receptor (PR), HER2, and Ki-67 status. The relevant cut-off value for high expression of Ki-67 was defined as 30\% described elsewhere [15]. Positive for HER2 is either IHC HER2 3+ (defined as uniform intense membrane staining of $>30 \%$ of invasive tumor cells) or fluorescence in situ hybridization (FISH) amplified (ratio of HER2 to CEP17 of $>2.2$ or average HER2 gene copy number $>6$ signals /nucleus for those test systems without an internal control probe according to ASCO/CAP guideline recommendations. As a result, $48 \mathrm{BC}$ specimens including 14 with HRPBC, 9 with HER2PBC, 11 HHPBC, and 14 TNBC were selected in this study. Additionally, HRPBC, HER2PBC, and HHPBC were categorized as any positive BC. The FFPE specimens that had more than $50 \%$ tumor content to be analyzed in this study were sectioned into 10 micrometers using a new blade and preserved in $1.5 \mathrm{~mL}$ Eppendorf tubes. Blade was changed for every tissue block to prevent the contamination of DNA.

\subsection{DNA Extraction and Purification}

Four or five unstained FFPE sections (1 mm thick) were deparaffinized and used for DNA extraction. DNA extraction and purification were performed using the RecoverAll Total Nucleic Acid Isolation Kit for FFPE (ThermoFisher Scientific, Waltham, MA, USA). Extracted DNA was quantified using Qubit 2.0 Fluorometer with Qubit dsDNA HS Assay kit and the TaqMan RNase P Detection Reagents kit (ThermoFisher Scientific, Waltham, MA, USA) and was considered appropriate when the nucleic acid concentration was $>10 \mathrm{ng} / \mu \mathrm{L}$.

\subsection{Library Preparation for DNA Panel}

DNA panel of the Ion Torrent Oncomine Comprehensive Assay (OCA) v3 (ThermoFisher Scientific) was used. The OCA v3 allows concurrent analysis of DNA and RNA to simultaneously detect multiple types of variants across 161 genes relevant to solid tumors, including hotspots, single nucleotide variants (SNVs), insertions and deletions 
(Indels), copy number variants (CNVs), and gene fusions, in a single workflow. Briefly, $20 \mathrm{ng}$ of the genomic DNA were used in two target amplification reactions, which were then combined. Library preparation for each specimen was performed using the Ion Ampliseq Library Kit 2.0 plus (ThermoFisher Scientific, Waltham, MA, USA) according to the manufacturer's instructions. The prepared libraries for the DNA panel were partially digested and phosphorylated using the FuPa reagent, ligated to different barcode adapters using the Ion Xpress Barcode Adapters 1-48 Kit (ThermoFisher Scientific, Waltham, MA, USA), then purified. The purified libraries were quantified using the Ion Library TaqMan Quantitation Kit (ThermoFisher Scientific, Waltham, MA, USA).

\subsection{Sequencing Analysis Using the Ion S5XL}

Pooled purified libraries of eight multiplexed tumor DNAs per 540 chip at a concentration of $50 \mathrm{pM}$ were used for chip loading on the Ion Chef with the Ion 540 chef Kit (ThermoFisher Scientific, Waltham, MA, USA) and subsequently sequenced on S5XL using Ion S540 chip (ThermoFisher Scientific, Waltham, MA, USA) as per the manufacturer's instructions.

\subsection{Sanger Sequencing}

Because somatic tumor alterations were identified without matched normal specimens, mutation origin with allele frequency of near $50 \%$ (heterozygous) or $100 \%$ (homozygous) as possible germline origin was determined by Sanger sequencing using matched germline DNA from peripheral blood. Additionally, Sanger sequencing was performed to confirm some of the detected alterations with mutant allele burden $>15 \%$. Capillary electrophoresis was performed on the 3730XL Genetic Analyzer (Applied Biosystems, Carlsbad, CA, USA). Sequence data were analyzed using the Sequencher DNA Sequence Analysis Software Demo Version 4.9 (Gene Codes Corporation, Ann Arbor, MI, USA).

\subsection{Bioinformatic Analysis}

Analysis of sequencing raw data was performed by Torrent Suite software ver 5.10 (ThermoFisher Scientific, Waltham, MA, USA) using default analysis parameters. Data analyses for variant calling (SNVs/multi-nucleotide variants [MNVs], indels, and CNVs) were performed in Ion Reporter software ver 5.10. Available online: https:/ /ionreporter. thermofisher.com/ir/ (accessed on 11 November 2020) with Torrent Variant Caller and Coverage Analysis plug-ins using default settings. A minimum sequencing depth of $500 \times$ was considered as adequate sequencing depth, and an allelic frequency of $5 \%$ was used as a cut-off for variants. Human genome build 19 was used as the reference for alignment. Briefly, annotation of the results, filtering of spurious and repeat errors, and interface for visualization of sequencing reads via Integrative Genome Viewer were performed using software built in-house, as previously described [16]. Mutations predicted to cause strong and moderate alteration on gene functions, such as stop gained/lost, initiator codon, missense, frameshift, and splice site mutation were manually reviewed by laboratory geneticists based on ACMG-AMP standards and guidelines [17]. Particularly, multiple missense functional predictors including SIFT, polyphen2, MutationTaster, and MutationAssessor were used to determine their deleterious effect. In addition, genes that play a role as either oncogene or tumor suppressor based on their typical behavior in cancer were assessed using ClinVar. Available online: https:/ /www.ncbi.nlm.nih.gov/clinvar/ (accessed on 11 November 2020), Online Mendelian Inheritance in Man. Available online: https://www.omim.org/ (accessed on 11 November 2020), and Catalogue of Somatic Mutations in Cancer. Available online: https:/ / cancer.sanger.ac.uk/cosmic (accessed on 11 November 2020). When the number of gene roles in cancer is more than half, it is defined as either oncogene or tumor suppressor dominance. On the other hand, data analyses for identifying copy number variations were performed in Ion Reporter software v5.10 with a baseline from the average sequencing depths achieved in a set of normal DNA from 16 peripheral blood specimens and manufacturer recommended settings. The copy numbers for genes in a given specimens were calculated by comparing the average sequencing depth 
achieved by the amplicons covering the gene in the specimen to the historical sequencing depths (baseline) by using the algorithm previously described [18].

\subsection{Statistical Analysis}

Descriptive statistics were used to show the mean \pm SD of age and BMI of studied population. To compare somatic alteration profiles between BC subtypes, genomic characteristics were compared across cohorts using one-way analysis of variance followed by Scheffe's post hoc test for continuous variables. The difference of somatic alterations was estimated using Fisher's exact test. A two-tailed $p<0.05$ was considered to indicate a statistically significant difference. Statistical analysis was performed using MedCalc Statistical Software Version 19.5.3 (MedCalc Software Ltd., Ostend, Belgium).

\section{Results}

\subsection{Clinicopathological Features of Studied Patients}

All 48 patients were female, and four had a family history of BC. The mean age \pm standard deviation (SD) at diagnosis was $62.4 \pm 11.2$ years (range, $43-81$ years). The mean body mass index was $24.7 \pm 3.6 \mathrm{~kg} / \mathrm{m}^{2}$. The majority of patients $(73 \%)$ had early-stage breast cancer (stage Ia, 42\% (20/48); stage IIa, 31\% (15/48)). All patients were followed up for up to 3 years. No clinical relapse or cancer-related death occurred during the follow-up period. The clinicopathological features of the patients are shown in Table 1.

Table 1. Comparison results of clinicopathological features in 48 Korean patients with breast cancer.

\begin{tabular}{|c|c|c|c|c|c|c|}
\hline Features & $\begin{array}{c}\text { Total } \\
(n=48)\end{array}$ & $\begin{array}{c}\text { HRPBC } \\
(n=14)\end{array}$ & $\begin{array}{c}\text { HER2PBC } \\
(n=9)\end{array}$ & $\begin{array}{c}\text { HHPBC } \\
(n=11)\end{array}$ & $\begin{array}{c}\text { TNBC } \\
(n=14)\end{array}$ & $p$ Value * \\
\hline Age (Mean $\pm S D)$, year & $62.4 \pm 11.2$ & $64.4 \pm 11.0$ & $63.6 \pm 10.7$ & $63.1 \pm 12.1$ & $59.1 \pm 11.4$ & 0.638 \\
\hline$<50$ & 7 & 1 & 1 & 1 & 4 & 0.365 \\
\hline$\geq 50$ & 41 & 13 & 8 & 10 & 10 & \\
\hline Familial history & 4 & 1 & 0 & 0 & 3 & 0.173 \\
\hline Postmenopause & 41 & 13 & 8 & 10 & 10 & 0.365 \\
\hline BMI $\left(\right.$ Mean \pm SD), $\mathrm{kg} / \mathrm{m}^{2}$ & $24.7 \pm 3.6$ & $24.9 \pm 3.0$ & $23.7 \pm 4.5$ & $24.8 \pm 4.0$ & $25.0 \pm 3.6$ & 0.846 \\
\hline Primary tumor size & & & & & & 0.402 \\
\hline 1 & 24 & 9 & 3 & 4 & 8 & \\
\hline 2 & 22 & 4 & 6 & 6 & 6 & \\
\hline 3 & 1 & 1 & 0 & 0 & 0 & \\
\hline 4 & 1 & 0 & 0 & 1 & 0 & \\
\hline Lymph node metastasis & & & & & & 0.601 \\
\hline 0 & 33 & 9 & 6 & 7 & 11 & \\
\hline 1 & 8 & 1 & 1 & 3 & 3 & \\
\hline 2 & 4 & 2 & 1 & 1 & 0 & \\
\hline 3 & 3 & 2 & 1 & 0 & 0 & \\
\hline Pathologic stage & & & & & & 0.214 \\
\hline I & 20 & 7 & 2 & 3 & 8 & \\
\hline II & 20 & 3 & 5 & 6 & 6 & \\
\hline III & 8 & 4 & 2 & 2 & 0 & \\
\hline $\mathrm{Ki67}, \%$ & & & & & & 0.004 \\
\hline$<30$ & 23 & 13 & 2 & 6 & 2 & \\
\hline$\geq 30$ & 25 & 1 & 7 & 5 & 12 & \\
\hline Type of breast surgery & & & & & & 0.590 \\
\hline Breast conservation & 29 & 10 & 4 & 6 & 9 & \\
\hline Total mastectomy & 19 & 4 & 5 & 5 & 5 & \\
\hline Type of axillary surgery & & & & & & 0.757 \\
\hline Sentinel node biopsy & 34 & 9 & 7 & 7 & 11 & \\
\hline Axillary dissection & 14 & 5 & 2 & 4 & 3 & \\
\hline Adjuvant chemotherapy & 40 & 8 & 9 & 9 & 14 & 0.009 \\
\hline
\end{tabular}


Table 1. Cont.

\begin{tabular}{|c|c|c|c|c|c|c|}
\hline Features & $\begin{array}{c}\text { Total } \\
(n=48)\end{array}$ & $\begin{array}{l}\text { HRPBC } \\
(n=14)\end{array}$ & $\begin{array}{c}\text { HER2PBC } \\
(n=9)\end{array}$ & $\begin{array}{c}\text { НHРBC } \\
(n=11)\end{array}$ & $\begin{array}{c}\text { TNBC } \\
(n=14)\end{array}$ & $p$ Value * \\
\hline Adjuvant radiotherapy & 36 & 13 & 5 & 7 & 11 & 0.168 \\
\hline Hormone therapy & 25 & 14 & 0 & 11 & 0 & $<0.001$ \\
\hline HER2 target therapy & 17 & 0 & 8 & 9 & 0 & $<0.001$ \\
\hline
\end{tabular}

HRPBC, hormone receptor positive breast cancer; HER2PBC, HER2 positive breast cancer; HHPBC, hormone receptor positive and HER2 positive breast cancer; TNBC, triple negative breast cancer; * $p$-value indicates statistical significance between TNBC and any positive BC by Fisher's exact test.

\subsection{Quality Control Metrics of Raw Sequencing Data}

In quality control $(\mathrm{QC})$ metrics for raw sequencing data generated from six independent experiments, the mean of total usable reads was $89,630,464(63.8 \%)$ and the mean of read length was $105 \mathrm{bp}$ (SD, 6; range, 96-111). The mean of mapped read count, on-target read rate, the mean depth of on-target regions, and uniformity were $11,299,984,95.4 \%$, $2976 \times$, and $93.3 \%$, respectively. Overall, all experiments satisfied the manufacturer's specifications ( $>95 \%$ of amplicons should have a read depth $>500 \times$ ).

\subsection{Somatic Alteration Profiles}

A total of 11,088 unfiltered variants were identified from the raw sequencing data using the OCA v3 DNA assay. We analyzed the filtered cancer driver genes to determine potential genes of interest. After variant filtering with Oncomine Comprehensive v3/w4.0/DNA/Single Sample workflow, 80 somatic SNVs or indels and 22 CNVs passed data analysis algorithms. Of the 80 somatic mutations, 37 nonsense, 35 missense, and 8 frameshift mutations were identified. Thirty nucleotide alterations ( 24 nonsense, 1 missense, and 5 frameshift) were newly identified. The p.His28Tyr of $M A X$ gene is predicted to be deleterious based on missense functional predictors (SIFT, deleterious (Score 0); Polyphen2, probably damaging (1.00); MutationTaster, disease causing (83); and MutationAssessor, high (3.57)). The eight most commonly altered genes were PIK3CA, TP53, $E R B B 2, B R C A 2, F A N C D 2, A K T 1, B R C A 1$, and FANCA (Figure 1). We examined genes altered in multiple specimens and found that three genes were altered in at least $20 \%$ of these specimens. PIK3CA was the most frequently altered gene, with variants found in 15 HRPBC or HHPBC specimens. The second most frequently altered gene was TP53, which was shared by 12 specimens. Interestingly, several oncogenic TP53 mutations in 6 out of 11 TNBCs, including p.Lys132Gln, p.Pro177_Cys182del, p.Gln192*, p.Glu204*, p.His214Arg, p.Tyr236Cys, p.Cys238*, p.Arg248Trp, p.Arg273His, and p.Pro278Leu were detected in 6 TNBC. We also found several recurrent mutations in other genes, including the previously reported mutations in p.Glu17Lys of AKT1 (2/3, 66\%), p.Lys812Argfs *3 of $B R C A 1$ (2/3, 66\%; germline), and p.Lys700Glu of SF3B1 (2/2, 100\%) (Supplementary Table S1). Meanwhile, of the 13 specimens, all $22 \mathrm{CNVs}$ in 10 different genes were amplified. Interestingly, $E R B B 2$, the third most frequently altered gene, was only amplified but not mutated in 10 HER2PBC or HHPBC specimens (Supplementary Table S2). We also found several CNVs in other genes: Two in CCND1, FGF3, and FGF19. One in AKT2, EGFR, ESR1, KIT, MYC, and PIK3CA.

Collectively, we found an average of 2.5 alterations per specimen (range, 1-8 alterations) in the 44 unique cancer driver genes (Figure 2). Twenty-five of the 41 specimens had an alteration in TP53 and/or PIK3CA. The most commonly recurring mutation was the p.His1047Arg of PIK3CA, which was found in the majority of the PIK3CA mutant specimens $(8 / 15,53 \%)$, followed by p.Asn345Lys $(2 / 15,13 \%)$ and p.Glu542Lys of PIK3CA $(2 / 15,13 \%)$. 


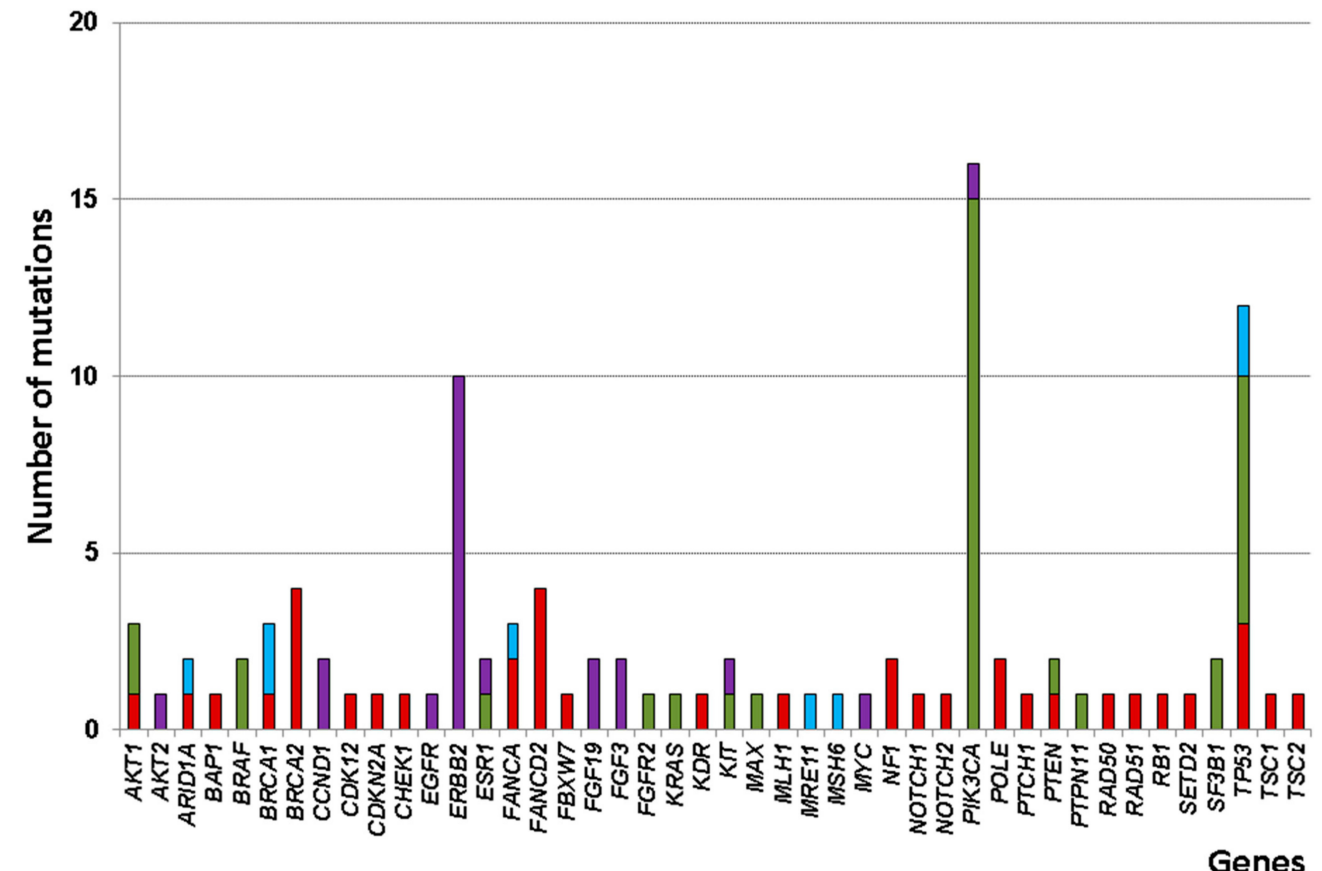

Figure 1. Frequencies of somatic alteration profiles in various genes identified using the Oncomine comprehensive v3 DNA assay in 41 patients with breast cancer. Genes are depicted on the x-axis, and the number of alterations is indicated on the y-axis. Green, missense mutation; red, nonsense mutation; blue, frameshift mutation; violet, amplification.

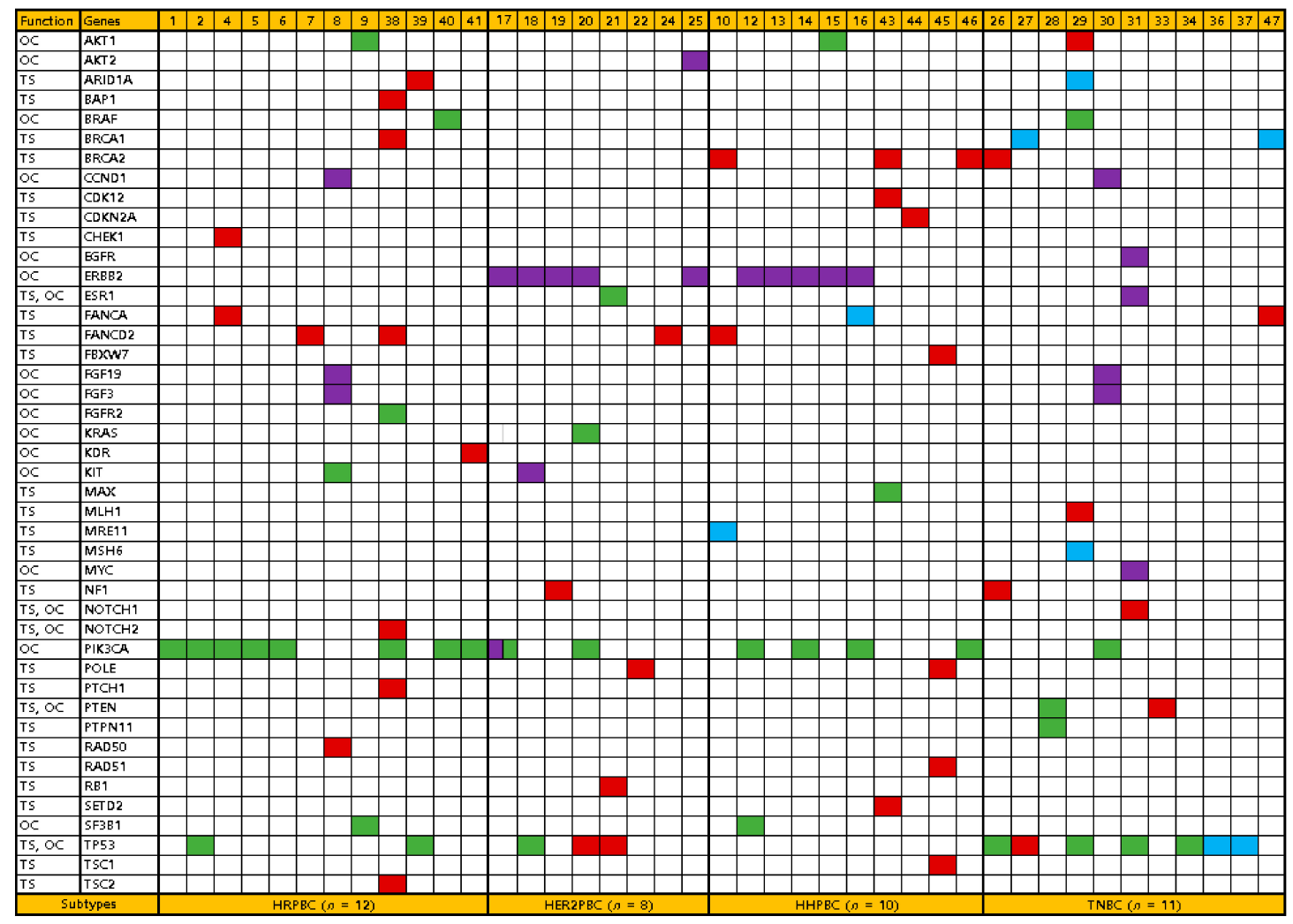

Figure 2. Distribution of somatic alteration profiles based on breast cancer subtypes in 41 patients with breast cancer. Each patient is depicted on the x-axis, and the genes are indicated on the y-axis. Green, missense mutation; red, nonsense mutation; blue, frameshift mutation; violet, amplification. HRPBC, hormone receptor-positive breast cancer; HER2PBC, hormone receptor-negative HER2-positive breast cancer; HHPBC, hormone receptor-positive and HER2-positive breast cancer; TNBC, triple-negative breast cancer. OC, oncogene; TS, tumor suppressor. 


\subsection{Comparison of Somatic Alteration Profiles between Breast Cancer Subtypes}

The presence of alteration and alteration type (nucleotide change vs. $\mathrm{CNV}$ ) were not statistically significant between TNBC and any positive BC. Of three frequent alterations, PIK3CA and TP53 alterations were statistically significant between TNBC and any positive $\mathrm{BC}$, respectively. Compared to the somatic alteration profiles of other $\mathrm{BC}$ subtypes, TNBC had significantly lower mutation frequency in PIK3CA (TNBC vs. HRPBC, $p=0.009$ ), but higher mutation frequency in TP53 (TNBC vs. HRPBC, $p=0.036$; TNBC vs. HHPBC, $p=0.004)$. Interestingly, significant differences in gene role dominance were observed between TNBC and HRPBC $(p=0.024)$ as well as between TNBC and any positive BC $(p=0.010)$ (Table 2).

Table 2. Comparison results of somatic alteration profiles in 48 Korean patients with breast cancer.

\begin{tabular}{|c|c|c|c|c|c|c|}
\hline Features & $\begin{array}{c}\text { Total } \\
(n=48)\end{array}$ & $\begin{array}{c}\text { HRPBC } \\
(n=14)\end{array}$ & $\begin{array}{c}\text { HER2PBC } \\
(n=9)\end{array}$ & $\begin{array}{c}\text { НHРBC } \\
(n=11)\end{array}$ & $\begin{array}{c}\text { TNBC } \\
(n=14)\end{array}$ & $p$ Value * \\
\hline Alteration number & & & & & & 0.942 \\
\hline 0 & 7 & 2 & 1 & 1 & 3 & \\
\hline 1 & 12 & 4 & 2 & 2 & 4 & \\
\hline$\geq 2$ & 29 & 8 & 6 & 8 & 7 & \\
\hline Alteration type & & & & & & 0.983 \\
\hline Missense & 35 & 15 & 5 & 7 & 8 & \\
\hline Nonsense & 37 & 12 & 6 & 11 & 8 & \\
\hline Frameshift & 8 & 0 & 0 & 2 & 6 & \\
\hline Amplification & 22 & 3 & 8 & 5 & 6 & \\
\hline \multicolumn{7}{|l|}{ Frequent alteration } \\
\hline$E R B B 2$ & 10 & 0 & 5 & 5 & 0 & 0.085 \\
\hline PIK3CA & 15 & 8 & 2 & 4 & 1 & 0.037 \\
\hline TP53 & 12 & 2 & 3 & 0 & 7 & 0.024 \\
\hline Gene role dominance & & & & & & 0.010 \\
\hline Oncogene & 17 & 7 & 4 & 5 & 1 & \\
\hline Tumor suppressor & 20 & 4 & 3 & 4 & 9 & \\
\hline Codominant & 4 & 1 & 1 & 1 & 1 & \\
\hline
\end{tabular}

HRPBC, hormone receptor positive breast cancer; HER2PBC, HER2 positive breast cancer; HHPBC, hormone receptor positive and HER2 positive breast cancer; TNBC, triple negative breast cancer; * $p$-value indicates statistical significance between TNBC and any positive BC by Fisher's exact test.

\subsection{Ki-67 Expression and PIK3CA/ TP53 Mutation Status}

TNBC showed frequently higher Ki-67 expression than any positive BC $(p=0.004)$ due to HRPBC $(p<0.001)$. Higher Ki-67 expression was not statistically different compared to HHPBC $(p=0.081)$ and HER2PBC $(p=1.000)$. PIK3CA and/or TP53 were the most commonly mutated gene in this study, with 25 of the 41 specimens $(61 \%)$ containing a mutation. To identify the roles of PIK3CA and/or TP53 and its association with Ki-67 expression, we subdefined TNBC with high Ki-67/unmutated PIK3CA/mutated TP53 subdivided from TNBC subtype. Six (specimens 26, 27, 31, 34, 36, and 37) of the 14 TNBC specimens showed high Ki-67/unmutated PIK3CA/mutated TP53 TNBC subtype. Interestingly, TNBC with high $\mathrm{Ki}-67 /$ unmutated PIK3CA/mutated TP53 appeared at a younger age (52.2 \pm 7.6 years) than any positive $\mathrm{BC}(63.7 \pm 11.0$ years). However, there was no significant difference in the age of onset between TNBC and each BC subtype or any positive BC. Furthermore, there was no association among PIK3CA and/or TP53 mutation status, Ki-67 expression, and cancer staging.

\section{Discussion}

Earlier onset, aggressive tumor phenotype, and more advanced stage at diagnosis are distinct features of TNBC in women with African ethnicity compared to Caucasians, denoting one of the most characteristic findings of racial disparity in cancer oncology [19]. However, the higher frequency of TNBC in African Americans is not related to a different 
genomic profile of commonly established tumor regulatory pathway genes [20]. In multiracial early onset TNBC study, African Americans had the highest number of deleterious mutations compared to European Americans, Hispanic, and Asian populations. However, the trend was reversed such that African Americans carried fewer mutations when focusing on mutations in the known breast cancer genes [21]. Thus, further classification of TNBC, considering ethnicity/genetic background, is required to in order to detect early neoplastic changes; thus, facilitating the detection of TNBC at an early stage is one of the most important challenges in the treatment of $\mathrm{BC}$. In this study, we demonstrate that the predictive potential of Ki-67, PIK3CA, and TP53 status in relatively early onset Korean $\mathrm{TNBC}$, compared to other $\mathrm{BC}$ subtypes. Combining somatic alteration profiles and Ki-67 state, TNBC with high Ki-67/unmutated PIK3CA/mutated TP53 appeared at a younger age than any positive $\mathrm{BC}$. Of eight Korean $\mathrm{BC}$ under the age of 50 years, four were high Ki-67/unmutated PIK3CA/mutated TP53 TNBC with stage 2A/B $(n=3)$ and $1 \mathrm{~A}(n=1)$. Ki-67 expression over $30 \%$ was significantly associated with worse prognosis, especially for stage I patients [15].

Similar to previous studies $[14,22,23]$, our study demonstrated that TP53 is the most commonly mutated gene, but PIK3CA mutation is rare in TNBC. In BC, oncogenic TP53 mutations in the DNA-binding domain from codon sequences 102 to 292 are related to poor prognosis compared to wild-type TP53, which is associated with better clinical outcome in BC [24]. However, the impact of oncogenic TP53 mutations on the overall survival and disease-free survival for TNBC was not available, because all patients were alive without disease recurrence during our 3-year follow-up period. A previous study reported that a subset of TNBC harbors somatic mutations in the genome repair system [25]. In our study, several tumor suppressor genes such as not only BRCA1/2 and TP53 but also MLH1, MSH6, NF1, and PTPN11 were predominantly altered in TNBC. However, there were no predominant mutated genes or hotspot mutations. A higher mutational burden of tumor was also more commonly observed in HR-negative BC than in HRPBC [26]. More deleterious mutations in multiple genes, including $B R C A 1, B R C A 2$ and other predisposition genes, are associated with TNBC [27]. In this study, deleterious alterations such as nonsense or frameshift were slightly high in TNBC $(57 \%, 8 / 14)$ than in any positive BC $(50 \%, 17 / 34)$, which were not statistically significant $(p=0.756)$. Similarly, multiple alterations with $\geqq 4$ in one specimen were observed in TNBC $(21 \%, 3 / 14)$ than in any positive BC $(15 \%, 5 / 34)$, which was not statistically significant $(p=0.676)$.

PIK3CA mutations are usually enriched in 29 to $45 \%$ of HR-positive tumors, with a lower frequency in TNBC [28]. Similar to other breast cancer studies, PIK3CA was the most commonly mutated oncogene $(31 \%, 15 / 48)$ in our study. The mutational incidence of PIK3CA in TNBC was much lower $(7 \%, 1 / 14)$ in this study than that in previously published research $[14,22,23]$. However, dysregulation of signaling through the PI3K and AKT signaling pathways is one of the most common oncogenic aberrations in TNBC. Selecting patients for AKT inhibition according to PI3KCA/AKT1/PTEN alterations appears to optimize the treatment outcomes [29].

Most TNBC patients do not carry germline mutations of $B R C A 1$ or $B R C A 2$, which are critical for maintaining genome integrity. However, pathological high-grade $B C$ s and TNBC often show somatic mutations or abnormal BRCA1 or BRCA2 expression [30]. In our study, two TNBC specimens with p.Lys812Argfs*3 germline BRCA1 mutation were identified. $\mathrm{BC}$ occurring in most germline $B R C A 1$ mutation carriers are TNBCs. In contrast, there is no specific breast cancer subtype in $B R C A 2$ carriers [31]. Defects in the genome repair machinery associated with $B R C A 1$ and $B R C A 2$ mutations could optimize the treatment outcomes of platinum-based chemotherapy or PARP inhibitors in these patients [32].

Alternative pathways in cell proliferation, differentiation, apoptosis, and invasion, such as paracrine/autocrine loops of growth factors, have been suggested as novel candidate therapeutic pathways and targets in TNBC. SHP-1/p-STAT3/VEGF-A axis is a potential therapeutic target for metastatic TNBC, and the more potent SC-78 may be a promising lead for suppressing metastasis of TNBC [33]. Sequential combination of do- 
cetaxel with a SHP-1 agonist enhanced suppression of p-STAT3 signaling and apoptosis in triple negative breast cancer cells [34]. Targeting SHP-1/p-STAT3 and the potential combination of SHP-1 agonist with chemotherapeutic docetaxel is a feasible therapeutic strategy for TNBC [35].

Our study had some limitations. First the number of studied patients was small, and the patients were from a single center, although each BC subtype was well classified. Second, comprehensive cancer panel sequencing was performed on only tumor DNA extracted from FFPE specimens. The quality of tumor DNA from FFPE is lower than that from fresh specimens, potentially causing variant call discrepancies [36]. A low tumor allele frequency below our cut-off of $5 \%$ carrying "actual" mutations could also be missed. Third, the result of targeted gene panel sequencing is affected by the genomic size targeted by the panel and by its gene composition. To resolve this discrepancy, core genes established to have diagnostic, therapeutic, or prognostic relevance in $\mathrm{BC}$ should be included when designing gene panels. Fourth, the short follow-up period of up to 3 years was inadequate to observe clinically meaningful associations between genomic alterations and clinicopathological features.

\section{Conclusions}

In conclusion, our study demonstrates that TNBC with high Ki-67/unmutated PIK3CA/ mutated TP53 may be related to relatively early-onset BC. The somatic alteration profiles from Korean TNBC patients were found to contribute to the mutation characteristics of Asian $\mathrm{BC}$ patients, providing insights into the genome landscape of $\mathrm{BC}$ and further evidence on the role of Ki-67, PIK3CA, and TP53 in breast carcinogenesis. These findings demonstrate the genomic heterogeneity between TNBC and other BC subtypes and could present a new approach for molecular targeted therapy in TNBC patients.

Supplementary Materials: The following are available online at https:/ /www.mdpi.com/2075-4 418/11/3/416/s1, Table S1: Details of mutation profiles in 39 Korean patients with breast cancer. Table S2: Details of copy number variations in 13 Korean patients with breast cancer.

Author Contributions: W.Y.S. contributed to the conception and design of the study, interpreted clinical medical record, and reviewed the draft. J.L. and B.K.K. interpreted clinical medical record. J.O.K. interpreted pathologic findings. J.P. performed the experiments, analyzed the molecular data, and drafted the manuscript. All authors have read and agreed to the published version of the manuscript.

Funding: The authors wish to acknowledge the financial support of the Catholic Medical Center Research Foundation made in the program year of 2020. This work was supported by The Catholic University of Korea Daejeon St. Mary's Hospital, Clinical research institute Grant funded by The Catholic University of Korea Daejeon St. Mary's Hospital (CMCDJ-P-2019-001).

Institutional Review Board Statement: This study protocol was approved by the Institutional Review Board of the Catholic University of Korea (No. DC20SASI0051; Date of approval: 30 June 2020). All subjects provided written informed consent for clinical and molecular analyses and publication before the study.

Informed Consent Statement: All subjects provided written informed consent for clinical and molecular analyses and publication before the study.

Data Availability Statement: The data presented in this study are available on request from the corresponding author.

Acknowledgments: The authors would like to thank Taekyu Lee (ThermoFisher Scientific Solutions, Seoul, Korea) for his contribution in providing technical support for Ion Torrent sequencing in this study.

Conflicts of Interest: The authors declare no conflict of interest. The funders had no role in the design of the study; in the collection, analyses, or interpretation of data; in the writing of the manuscript, or in the decision to publish the results. 


\section{References}

1. Bray, F.; Ferlay, J.; Soerjomataram, I.; Siegel, R.L.; Torre, L.A.; Jemal, A. Global cancer statistics 2018: GLOBOCAN estimates of incidence and mortality worldwide for 36 cancers in 185 countries. CA Cancer J. Clin. 2018, 68, 394-424. [CrossRef] [PubMed]

2. Kang, S.Y.; Kim, Y.S.; Kim, Z.; Kim, H.Y.; Kim, H.J.; Park, S.; Bae, S.Y.; Yoon, K.H.; Lee, S.B.; Lee, S.K.; et al. Breast Cancer Statistics in Korea in 2017: Data from a Breast Cancer Registry. J. Breast Cancer 2020, 23, 115-128. [CrossRef]

3. Loibl, S.; Treue, D.; Budczies, J.; Weber, K.; Stenzinger, A.; Schmitt, W.D.; Weichert, W.; Jank, P.; Furlanetto, J.; Klauschen, F.; et al. Mutational Diversity and Therapy Response in Breast Cancer: A Sequencing Analysis in the Neoadjuvant GeparSepto Trial. Clin. Cancer Res. 2019, 25, 3986-3995. [CrossRef] [PubMed]

4. Early Breast Cancer Trialists' Collaborative Group. Effects of chemotherapy and hormonal therapy for early breast cancer on recurrence and 15-year survival: An overview of the randomised trials. Lancet 2005, 365, 1687-1717. [CrossRef]

5. Lehmann, B.D.; Jovanović, B.; Chen, X.; Estrada, M.V.; Johnson, K.N.; Shyr, Y.; Moses, H.L.; Sanders, M.E.; Pietenpol, J.A. Refinement of Triple-Negative Breast Cancer Molecular Subtypes: Implications for Neoadjuvant Chemotherapy Selection. PLOS ONE 2016, 11, e0157368. [CrossRef] [PubMed]

6. Golubnitschaja, O.; Filep, N.; Yeghiazaryan, K.; Blom, H.J.; Hofmann-Apitius, M.; Kuhn, W. Multi-omic approach decodes paradoxes of the triple-negative breast cancer: Lessons for predictive, preventive and personalised medicine. Amino Acids 2018, 50, 383-395. [CrossRef] [PubMed]

7. Turner, N.C.; Reis-Filho, J.S. Basal-like breast cancer and the BRCA1 phenotype. Oncogene 2006, 25, 5846-5853. [CrossRef]

8. Turner, N.; Tutt, A.; Ashworth, A. Hallmarks of 'BRCAness' in sporadic cancers. Nat. Rev. Cancer 2004, 4, 814-819. [CrossRef]

9. Nik-Zainal, S.; Davies, H.; Staaf, J.; Ramakrishna, M.; Glodzik, D.; Zou, X.; Martincorena, I.; Alexandrov, L.B.; Martin, S.; Wedge, D.C.; et al. Landscape of somatic mutations in 560 breast cancer whole-genome sequences. Nature 2016, 534, 47-54. [CrossRef]

10. Robinson, D.R.; Wu, Y.M.; Lonigro, R.J.; Vats, P.; Cobain, E.; Everett, J.; Cao, X.; Rabban, E.; Kumar-Sinha, C.; Raymond, V.; et al. Integrative clinical genomics of metastatic cancer. Nature 2017, 548, 297-303. [CrossRef]

11. Bianchini, G.; Balko, J.M.; Mayer, I.A.; Sanders, M.E.; Gianni, L. Triple-negative breast cancer: Challenges and opportunities of a heterogeneous disease. Nat. Rev. Clin. Oncol. 2016, 13, 674-690. [CrossRef] [PubMed]

12. Geenen, J.J.J.; Linn, S.C.; Beijnen, J.H.; Schellens, J.H.M. PARP Inhibitors in the Treatment of Triple-Negative Breast Cancer. Clin. Pharmacokinet. 2018, 57, 427-437. [CrossRef] [PubMed]

13. Chen, L.; Li, C.I. Racial disparities in breast cancer diagnosis and treatment by hormone receptor and HER2 status. Cancer Epidemiol. Biomark. Prev. 2015, 24, 1666-1672. [CrossRef] [PubMed]

14. Niyomnaitham, S.; Parinyanitikul, N.; Roothumnong, E.; Jinda, W.; Samarnthai, N.; Atikankul, T.; Suktitipat, B.; Thongnoppakhun, W.; Limwongse, C.; Pithukpakorn, M. Tumor mutational profile of triple negative breast cancer patients in Thailand revealed distinctive genetic alteration in chromatin remodeling gene. PeerJ 2019, 7, e6501. [CrossRef]

15. Zhu, X.; Chen, L.; Huang, B.; Wang, Y.; Ji, L.; Wu, J.; Di, G.; Liu, G.; Yu, K.; Shao, Z.; et al. The prognostic and predictive potential of Ki-67 in triple-negative breast cancer. Sci. Rep. 2020, 10, 225. [CrossRef]

16. Singh, R.R.; Patel, K.P.; Routbort, M.J.; Reddy, N.G.; Barkoh, B.A.; Handal, B.; Kanagal-Shamanna, R.; Greaves, W.O.; Medeiros, L.J.; Aldape, K.D.; et al. Clinical validation of a next-generation sequencing screen for mutational hotspots in 46 cancer-related genes. J. Mol. Diagn. 2013, 15, 607-622. [CrossRef]

17. Richards, S.; Aziz, N.; Bale, S.; Bick, D.; Das, S.; Gastier-Foster, J.; Grody, W.W.; Hegde, M.; Lyon, E.; Spector, E.; et al. Standards and guidelines for the interpretation of sequence variants: A joint consensus recommendation of the American College of Medical Genetics and Genomics and the Association for Molecular Pathology. Genet. Med. 2015, 17, 405-424. [CrossRef]

18. Singh, R.R.; Patel, K.P.; Routbort, M.J.; Aldape, K.; Lu, X.; Manekia, J.; Abraham, R.; Reddy, N.G.; Barkoh, B.A.; Veliyathu, J.; et al. Clinical massively parallel next-generation sequencing analysis of 409 cancer-related genes for mutations and copy number variations in solid tumours. Br. J. Cancer 2014, 111, 2014-2023. [CrossRef]

19. Siddharth, S.; Sharma, D. Racial Disparity and Triple-Negative Breast Cancer in African-American Women: A Multifaceted Affair between Obesity, Biology, and Socioeconomic Determinants. Cancers 2018, 10, 514. [CrossRef]

20. Ademuyiwa, F.O.; Tao, Y.; Luo, J.; Weilbaecher, K.; Ma, C.X. Differences in the mutational landscape of triple-negative breast cancer in African Americans and Caucasians. Breast Cancer Res. Treat. 2017, 161, 491-499. [CrossRef]

21. Liu, Q.; Yao, S.; Zhao, H.; Hu, Q.; Kwan, M.L.; Roh, J.M.; Ambrosone, C.B.; Kushi, L.H.; Liu, S.; Zhu, Q. Early-onset triple-negative breast cancer in multiracial/ethnic populations: Distinct trends of prevalence of truncation mutations. Cancer Med. 2019, 8, 1845-1853. [CrossRef] [PubMed]

22. Lefebvre, C.; Bachelot, T.; Filleron, T.; Pedrero, M.; Campone, M.; Soria, J.C.; Massard, C.; Lévy, C.; Arnedos, M.; Lacroix-Triki, M.; et al. Mutational Profile of Metastatic Breast Cancers: A Retrospective Analysis. PLoS Med. 2016, 13, e1002201. [CrossRef] [PubMed]

23. Uscanga-Perales, G.I.; Santuario-Facio, S.K.; Sanchez-Dominguez, C.N.; Cardona-Huerta, S.; Muñoz-Maldonado, G.E.; RuizFlores, P.; Barcenas-Walls, J.R.; Osuna-Rosales, L.E.; Rojas-Martinez, A.; Gonzalez-Guerrero, J.F.; et al. Genetic alterations of triple negative breast cancer (TNBC) in women from Northeastern Mexico. Oncol. Lett. 2019, 17, 3581-3588. [CrossRef] [PubMed]

24. Soussi, T.; Wiman, K.G. TP53: An oncogene in disguise. Cell Death Differ. 2015, 22, 1239-1249. [CrossRef] [PubMed]

25. Stephens, P.J.; Tarpey, P.S.; Davies, H.; Van Loo, P.; Greenman, C.; Wedge, D.C.; Nik-Zainal, S.; Martin, S.; Varela, I.; Bignell, G.R.; et al. The landscape of cancer genes and mutational processes in breast cancer. Nature 2012, 486, 400-404. [CrossRef] [PubMed] 
26. Barrett, M.T.; Lenkiewicz, E.; Malasi, S.; Basu, A.; Yearley, J.H.; Annamalai, L.; McCullough, A.E.; Kosiorek, H.E.; Narang, P.; Wilson Sayres, M.A.; et al. The association of genomic lesions and PD-1/PD-L1 expression in resected triple-negative breast cancers. Breast Cancer Res. 2018, 20, 71. [CrossRef]

27. Shi, Y.; Jin, J.; Ji, W.; Guan, X. Therapeutic landscape in mutational triple negative breast cancer. Mol. Cancer 2018, 17, 99. [CrossRef] [PubMed]

28. Mayor, S. Comprehensive molecular portraits of human breast tumours. Nature 2012, 490, 61-70. [CrossRef]

29. Pascual, J.; Turner, N.C. Targeting the PI3-kinase pathway in triple-negative breast cancer. Ann. Oncol. 2019, 30, 1051-1060. [CrossRef]

30. Von Wahlde, M.K.; Timms, K.M.; Chagpar, A.; Wali, V.B.; Jiang, T.; Bossuyt, V.; Saglam, O.; Reid, J.; Gutin, A.; Neff, C.; et al. Intratumor Heterogeneity of Homologous Recombination Deficiency in Primary Breast Cancer. Clin. Cancer Res. 2017, 23, 1193-1199. [CrossRef]

31. Atchley, D.P.; Albarracin, C.T.; Lopez, A.; Valero, V.; Amos, C.I.; Gonzalez-Angulo, A.M.; Hortobagyi, G.N.; Arun, B.K. Clinical and pathologic characteristics of patients with BRCA-positive and BRCA-negative breast cancer. J. Clin. Oncol. 2008, 26, 4282-4288. [CrossRef] [PubMed]

32. Papadimitriou, M.; Mountzios, G.; Papadimitriou, C.A. The role of PARP inhibition in triple-negative breast cancer: Unraveling the wide spectrum of synthetic lethality. Cancer Treat. Rev. 2018, 67, 34-44. [CrossRef] [PubMed]

33. Su, J.C.; Mar, A.C.; Wu, S.H.; Tai, W.T.; Chu, P.Y.; Wu, C.Y.; Tseng, L.M.; Lee, T.C.; Chen, K.F.; Liu, C.Y.; et al. Disrupting VEGF-A paracrine and autocrine loops by targeting SHP-1 suppresses triple negative breast cancer metastasis. Sci. Rep. 2016, 6, 28888. [CrossRef] [PubMed]

34. Liu, C.Y.; Chen, K.F.; Chao, T.I.; Chu, P.Y.; Huang, C.T.; Huang, T.T.; Yang, H.P.; Wang, W.L.; Lee, C.H.; Lau, K.Y.; et al. Sequential combination of docetaxel with a SHP-1 agonist enhanced suppression of p-STAT3 signaling and apoptosis in triple negative breast cancer cells. J. Mol. Med. 2017, 95, 965-975. [CrossRef] [PubMed]

35. Liu, C.Y.; Su, J.C.; Huang, T.T.; Chu, P.Y.; Huang, C.T.; Wang, W.L.; Lee, C.H.; Lau, K.Y.; Tsai, W.C.; Yang, H.P.; et al. Sorafenib analogue SC-60 induces apoptosis through the SHP-1/STAT3 pathway and enhances docetaxel cytotoxicity in triple-negative breast cancer cells. Mol. Oncol. 2017, 11, 266-279. [CrossRef] [PubMed]

36. Chalmers, Z.R.; Connelly, C.F.; Fabrizio, D.; Gay, L.; Ali, S.M.; Ennis, R.; Schrock, A.; Campbell, B.; Shlien, A.; Chmielecki, J.; et al. Analysis of 100,000 human cancer genomes reveals the landscape of tumor mutational burden. Genome Med. 2017, 9, 34. [CrossRef] 